hep-th/0612xxx

FIT HE - 06-03

KYUSHU-HET **

Kagoshima HE - 06-3

\title{
Flavor quarks in $\mathrm{AdS}_{4}$ and gauge/gravity correspondence
}

\author{
Kazuo Ghorokut, Masafumi Ishiharat, Akihiro Nakamuras \\ ${ }^{\dagger}$ Fukuoka Institute of Technology, Wajiro, Higashi-ku \\ Fukuoka 811-0295, Japan \\ ${ }^{\ddagger}$ Department of Physics, Kyushu University, Hakozaki, Higashi-ku \\ Fukuoka 812-8581, Japan \\ $\S$ Department of Physics, Kagoshima University, Korimoto \\ 1-21-35,Kagoshima 890-0065, Japan
}

\begin{abstract}
The non-perturbative properties of the gauge theories in the $\mathrm{AdS}_{4}$ are studied in the dual supergravity by including light flavor quarks, which are introduced by a D7 brane embedding. Contrary to the cases of Minkowski and $\mathrm{dS}_{4}$, the dilaton does not play any important dynamical role in the $\mathrm{AdS}_{4}$ case, and the characteristic properties like the quark confinement and the chiral symmetry breaking are realized mainly due to the geometry $\mathrm{AdS}_{4}$. The possible hadron spectra are also examined, and we find that the meson spectra are well described by the formula given by the field theory in $\mathrm{AdS}_{4}$, but the characteristic mass scale is modified by the gauge interactions for exited states.
\end{abstract}

\footnotetext{
${ }^{\dagger}$ gouroku@dontaku.fit.ac.jp

${ }^{\ddagger}$ masafumi@higgs.phys.kyushu-u.ac.jp

${ }^{\S}$ nakamura@sci.kagoshima-u.ac.jp
} 


\section{Introduction}

Recently, based on the gauge/gravity correspondence [1], many non-perturbative properties of Yang-Mills theories with quarks have been uncovered in terms of the superstring theory [2]-[11]. In these approaches, the flavor quarks are introduced by embedding probe D7 brane(s) in an appropriate bulk 10d background, which could describe the QCD type of Yang-Mills theory, and many successful results have been obtained for the properties of quarks and their bound states.

Up to now, almost all efforts have been devoted to the study of the gauge theory in the $4 \mathrm{~d}$ Minkowski space-time. Previously, we extended our analysis to the gauge theory in $4 \mathrm{~d}$ de Sitter space, $\mathrm{dS}_{4}$, and many interesting properties are found [12, 13. Here we furthermore extend the analysis into the case of $4 \mathrm{~d}$ anti-de Sitter space-time, $\mathrm{AdS}_{4}$, which is embedded in the $10 \mathrm{~d}$ bulk. The quantum field theory in the $\mathrm{AdS}_{4}$ has been studied in [14, and the discrete mass spectra of scalar field has been found under the reflective boundary conditions for the universal covering space CAdS. The same mass formula for the massive spin two states, which corresponds to the glueball states in the gauge theory, are found from $5 \mathrm{~d}$ AdS analysis by demanding the normalizability of the wave-function in the fifth direction [15]. This implies the usefulness of the holographic approach to the gauge theory in the $\mathrm{AdS}_{4}$. In [15], the existence of the massive graviton in $\mathrm{AdS}_{4}$ has been pointed out in a three brane where gravity couples with CFT. And this observation has been further studied by Porrati as a "Higgs phenomena" driven by dynamics in the geometry $\mathrm{AdS}_{4}$ [16, 17].

Here we concentrate our attention on the properties of flavor quarks which couple to the CFT embedded in $\mathrm{AdS}_{4}$, which would give a strong constraint on the dynamics of quarks interacting with deformed CFT as seen in the case of the gravity. The analysis is performed according to the formulation given before for the case of $\mathrm{dS}_{4}$ [12].

In section 2, we give the setting of our model for the present study. In section 3, the potential between quark and anti-quark and the effective quark mass are studied through the Wilson Polyakov loop estimate. In section 4, the embedding of the D7brane and the chiral symmetry breaking are discussed. In section 5 , the possible bound state for the meson and baryon are discussed. The meson spectra are compared with the formula given by the field theory in $\mathrm{AdS}_{4}$. The summary is given in the final section.

\section{Background geometry}

We start from the type IIB supergravity with the following bosonic action,

$$
S=\frac{1}{2 \kappa^{2}} \int d^{10} x \sqrt{-g}\left(R-\frac{1}{2}(\partial \Phi)^{2}+\frac{1}{2} e^{2 \Phi}(\partial \chi)^{2}-\frac{1}{4 \cdot 5 !} F_{(5)}^{2}\right),
$$

where other fields are neglected since we need not them, and $\chi$ is Wick rotated. By taking the ansatz for $F_{(5)}, F_{m_{1} \cdots m_{5}}=-\sqrt{\Lambda} / 2 \epsilon_{m_{1} \cdots m_{5}}$ and $F_{\alpha_{1} \cdots \alpha_{5}}=\sqrt{\Lambda} / 2 \epsilon_{\alpha_{1} \cdots \alpha_{5}}$ [18, 19, 20], and for the 10d metric as $M_{5} \times S^{5}$ or $d s^{2}=g_{m n} d x^{m} d x^{n}+g_{\alpha \beta} d x^{\alpha} d x^{\beta}$ *,

\footnotetext{
${ }^{*}$ Here $m, n$ denote $\mu, \nu=0 \sim 3$ and the direction of $r$, the fifth coordinate. And $\alpha, \beta=5 \sim 9$.
} 
the equations of motion are given as

$$
\begin{gathered}
R_{m n}=-\Lambda g_{m n}+\frac{1}{2} \partial_{m} \Phi \partial_{n} \Phi-\frac{1}{2} e^{2 \Phi} \partial_{m} \chi \partial_{n} \chi \\
\frac{1}{\sqrt{-g}} \partial_{m}\left(\sqrt{-g} g^{m n} \partial_{n} \Phi\right)=-e^{2 \Phi} \partial_{m} \chi \partial_{n} \chi g^{m n} \\
\frac{1}{\sqrt{-g}} \partial_{m}\left(\sqrt{-g} g^{m n} e^{2 \Phi} \partial_{n} \chi\right)=0, \\
R_{\alpha \beta}=\Lambda g_{\alpha \beta} .
\end{gathered}
$$

Using the ansatz,

$$
\chi=-e^{-\Phi}+\chi_{0}
$$

where $\chi_{0}$ is a constant, the equation (2) is written as

$$
R_{m n}=-\Lambda g_{m n}
$$

We notice that this equation (7) is independent of the fields $\Phi$ and $\chi$. This implies the usual 5d AdS solution, and we know that the four dimensional slice perpendicular to the fifth coordinate $r$ is taken as $\mathrm{AdS}_{4}$ as well as Minkowski or $\mathrm{dS}_{4}$. Here we consider the case of $\mathrm{AdS}_{4}$ embedded in $\mathrm{AdS}_{5}$.

After adopting the solution $\mathrm{AdS}_{4}$ embedded in $\mathrm{AdS}_{5}$ for the metric, $\Phi$ and $\chi$ are obtained from (3) and (6). Thus we obtain the following solution,

$$
\begin{gathered}
d s_{10}^{2}=G_{M N} d X^{M} d X^{N} \\
=e^{\Phi / 2}\left\{\frac{r^{2}}{R^{2}} A^{2}\left(-d t^{2}+a(t)^{2} \gamma(x)^{2}\left(d x^{i}\right)^{2}\right)+\frac{R^{2}}{r^{2}} d r^{2}+R^{2} d \Omega_{5}^{2}\right\}, \\
e^{\Phi}=1+\frac{q}{6}\left(1-\frac{1-\left(r_{0} / r\right)^{2}}{\left(1+\left(r_{0} / r\right)^{2}\right)}\left[1+\frac{2\left(r_{0} / r\right)^{2}}{\left(1+\left(r_{0} / r\right)^{2}\right)^{2}}\right]\right), \quad \chi=-e^{-\Phi}+\chi_{0}, \\
A=1+\left(\frac{r_{0}}{r}\right)^{2}, \quad a(t)=\frac{R^{2}}{2 r_{0}} \sin \left(2 \frac{r_{0}}{R^{2}} t\right), \quad \gamma(x)=\frac{1}{1-x_{i} x^{i} /\left(4 \tilde{r}_{0}^{2}\right)},
\end{gathered}
$$

where $M, N=0 \sim 9, R=\sqrt{\Lambda} / 2=\left(4 \pi N_{c}\right)^{1 / 4}$ and $\tilde{r}_{0}$ is an arbitrary scale factor. We comment on other two integration constants, $r_{0}$ and $q$. First, $r_{0}$ has nothing to do with the horizon since there is no horizon in the present solution and $r$ is considered in the all region of $0<r<\infty$ contrary to the $\mathrm{dS}_{4}$ case. This parameter $r_{0}$ is however important since it is related to the $4 \mathrm{~d}$ cosmological constant $\lambda$, which is negative, as follows

$$
-\lambda=4 \frac{r_{0}^{2}}{R^{4}}
$$

And $q$ is a constant which corresponds to the gauge fields condensate [10] defined as the vacuum expectation value (VEV) of gauge field squared as,

$$
q \equiv \tilde{q} / r_{0}^{4}=\left\langle F_{\mu \nu}^{2}\right\rangle / r_{0}^{4}
$$


We assume that $\left\langle F_{\mu \nu}^{2}\right\rangle=\tilde{q}$ is finite, and other field configurations are set to be zero.

Our model is based on type IIB supergravity, and we solved the equations of motion with dilaton and axion with the ansatz (6). In this case, the condition for the supersymmetry of the solution is reduced to the presence of the Killing spinor $u$ [18, 20] which satisfies the following equation in terms of the $10 \mathrm{~d}$ gamma matrices $\Gamma_{M}$,

$$
\delta \Psi_{M}=\left(D_{M}-\frac{\sqrt{\Lambda}}{4} \Gamma_{M}\right) u=0,
$$

where $\Psi_{M}$ denotes the gravitino and $D_{M}=\partial_{M}+\frac{1}{4} \omega_{M}^{A B} \Gamma_{A} \Gamma_{B}$. The equation for the dilatino, $\delta \lambda=0$, is satisfied due to the ansatz (6). The $S^{5}$ part of (13) is obtained as in the case of $\mathrm{AdS}_{5} \times S^{5}$ [21]. For the $\mathrm{AdS}_{5}$ part the condition is modified due to the metric of $\mathrm{AdS}_{4}$. In this case, the covariant derivatives are written as,

$$
\begin{aligned}
D_{r} u= & \partial_{r} u \\
D_{0} u= & \left(\partial_{0}-\frac{\mu}{2}\left(\frac{1-\left(r_{0} / r\right)^{2}}{1+\left(r_{0} / r\right)^{2}}\right) \Gamma_{0} \Gamma_{r}\right) u \\
D_{i} u= & \left(\partial_{i}-\frac{\gamma(x)}{2} \partial_{0} a(t) \hat{\Gamma}_{i} \hat{\Gamma}_{0}+\frac{1}{2 \gamma(x)} \partial_{j} \gamma(x) \hat{\Gamma}_{i} \hat{\Gamma}_{j}\right. \\
& \left.-\frac{\mu}{2 a(t) \gamma(x)}\left(\frac{1-\left(r_{0} / r\right)^{2}}{1+\left(r_{0} / r\right)^{2}}\right) \Gamma_{i} \Gamma_{r}\right) u
\end{aligned}
$$

where $\hat{\Gamma}_{M}$ denotes the local Lorentz constant gamma matrix. From these, we find easily, for finite $r_{0}$, that there is no Killing spinor, the solution of (13), of the form $u=$ $f\left(y, x^{\mu}\right) u_{0}$, where $u_{0}$ is some constant spinor. In other words, there is no supersymmetry in the present case. We notice that the supersymmetry is also broken by our D7 brane embedding since the $\kappa$ symmetry of the D7 brane action is lost [10] when the chiral symmetry is broken.

In this model, the four dimensional slice represents the $\mathrm{AdS}_{4}$ universe characterized by the $4 \mathrm{~d}$ cosmological constant $\lambda$. Then the bulk gravity describes the gauge theory in the $\mathrm{AdS}_{4}$. The gauge coupling constant in the present model is finite in the both limits of ultraviolet and infrared,

$$
g_{\mathrm{YM}}^{2}=e^{\Phi}=\left\{\begin{array}{ll}
1+\frac{q}{3}-q\left(\frac{r}{r_{0}}\right)^{4} & \text { for } r \rightarrow 0 \text { (IR limit) } \\
1+q\left(\frac{r_{0}}{r}\right)^{4} & \text { for } r \rightarrow \infty \text { (UV limit) }
\end{array} .\right.
$$

Meanwhile, in the limit of Minkowski space or the limit of $r_{0} \rightarrow 0$, we have $g_{\mathrm{YM}}^{2}=1+\frac{\tilde{q}}{r^{4}}$, which is equivalent to the supersymmetric solution given previously [10]. In this case, quark is confined since the YM coupling constant becomes very strong in the infrared limit, $r \rightarrow 0$ for $\tilde{q}>0$. But, for finite $r_{0}$ or in the $\mathrm{AdS}_{4}$ space-time, in the limit $r \rightarrow 0$, we find $g_{\mathrm{YM}}^{2} \rightarrow 1+\frac{q}{3}$, and it is not enough large for the quark confinement. However, the confinement is seen in this case due to the behavior of the warp factor $A(r)$ since it diverges at $r=0$ for finite $r_{0}$. This is assured by the calculation of the Wilson loop. Then the result for the quark confinement is independent of the value of $q$, which is needed in the Minkowski space-time for the quark confinement, in the $\mathrm{AdS}_{4}$. 


\section{$3 \quad$ Wilson Loop and Quark Confinement}

We study here the quark confinement through the Wilson-Polyakov loop in $S U(N)$ gauge theory defined as, $W=\frac{1}{N} \operatorname{Tr} P e^{i \int A_{0} d t}$. Then the quark-antiquark potential $V_{q \bar{q}}$ is derived from the expectation value of a parallel Wilson-Polyakov loop. From the dual gravity side, it is represented as

$$
\langle W\rangle \sim e^{-S}
$$

in terms of the Nambu-Goto action

$$
S=-\frac{1}{2 \pi \alpha^{\prime}} \int d \tau d \sigma \sqrt{-\operatorname{det} h_{a b}}
$$

with the induced metric $h_{a b}=G_{\mu \nu} \partial_{a} X^{\mu} \partial_{b} X^{\nu}$. The string world-sheet is parameterized by $\sigma, \tau$.

We examine quark-antiquark potentials in the background given above. To study possible static string configurations of a pair of quark and anti-quark, we choose as $X^{0}=t=\tau$ and $X^{1}=x^{1}=\sigma$, then the Nambu-Goto Lagrangian in the background (8) becomes

$$
L_{\mathrm{NG}}=-\frac{1}{2 \pi \alpha^{\prime}} \int d \sigma e^{\Phi / 2} A(r) \sqrt{r^{\prime 2}+\left(\frac{r}{R}\right)^{4}(A(r) a(t) \gamma(x))^{2}},
$$

where the prime denotes the derivative with respect to $\sigma$. The test string has two possible configurations: (i) a pair of parallel string, which connects the D7 and D3 branes, and (ii) a U-shaped string whose two end-points are on the D7 brane.

First, we study the configuration (i). In this case, the parallel strings have no correlation each other, then the total energy of this configuration is two times of one effective quark mass, $\tilde{m}_{q}$. It is given by a string configuration which stretches between $r=0$ and $r_{\max }$, which denote the position of D3 and D7 branes respectively. So we can take as $r=\sigma$, then we obtain

$$
\tilde{m}_{q}=\frac{1}{2 \pi \alpha^{\prime}} \int_{0}^{r_{\max }} d r e^{\Phi / 2} A(r)
$$

Near $r=0$, the integrand is approximated as $e^{\Phi / 2} A(r) \sim \sqrt{1+\frac{q}{3}}\left(\frac{r_{0}}{r}\right)^{2}$, then we find that $\tilde{m}_{q}$ diverges as

$$
\left.\tilde{m}_{q} \sim \sqrt{1+\frac{q}{3}} \frac{r_{0}^{2}}{r}\right|_{r \rightarrow 0} \rightarrow \infty .
$$

Thus the configuration of (i) can not be realized. In other words, the quark should confined. And this result is satisfied even if $q=0$, then the confinement is caused only by the gravitational effect.

The next check of the quark confinement is to see the area law through the U-shaped configuration. The energy of this configuration is obtained from (20) as

$$
E=-L_{\mathrm{NG}}=\frac{1}{2 \pi \alpha^{\prime}} \int d \tilde{\sigma} e^{\Phi / 2} A(r) \sqrt{\left(\partial_{\tilde{\sigma}} r\right)^{2}+\left(\frac{r}{R}\right)^{4}(A(r))^{2}}
$$




$$
=\frac{1}{2 \pi \alpha^{\prime}} \int d \tilde{\sigma} n \sqrt{1+\left(\frac{R^{2}}{r^{2} A} \partial_{\tilde{\sigma}} r\right)^{2}}
$$

where

$$
n=e^{\Phi / 2}\left(\frac{r A}{R}\right)^{2}
$$

and

$$
\tilde{\sigma}=a(t) \int d \sigma \gamma(\sigma)=a(t) \int d \sigma \frac{1}{1-\sigma^{2} / 4} .
$$

Here the coordinates of the string action are set as $x^{1}=\sigma, x^{2}=x^{3}=0$. In this case, we obtain

$$
\tilde{\sigma}=4 a(t) \tanh ^{-1}\left(\tan \left(\frac{\theta}{2}\right)\right), \quad \sigma=2 \cos (\theta),
$$

where $0 \leq \theta<\pi / 2$, and we find $0 \leq \tilde{\sigma}<\infty$. The physical distance between the quark and anti-quark is measured by $\tilde{\sigma}$ rather than $\sigma$ which is restricted as $-2<\sigma<2$ in our definition of the metric given by (8) .

Then the distance between the quark and the anti-quark at $t=0$ is given as

$$
\tilde{L}=2 \int_{\tilde{\sigma}_{\min }}^{\tilde{\sigma}_{\max }} d \tilde{\sigma}=2\left(\tilde{\sigma}_{\min }-\tilde{\sigma}_{\max }\right)
$$

where $\tilde{\sigma}_{\min }=\tilde{\sigma}\left(r_{\min }\right), \tilde{\sigma}_{\max }=\tilde{\sigma}\left(r_{\max }\right)$, and $r_{\min }$ is determined as $\left.\partial_{\tilde{\sigma}} r\right|_{r_{\min }}=0$. So $r_{\min }$ gives a midpoint of the U-shaped string. On the other hand, $r_{\max }$ is given by the position of the D7 brane, the end points of the U-shaped string.

Before giving the numerical estimation of the Wilson loop, we give an analytic approximate estimation according to the method given by Gubser [22]. The Eq. (24) can be approximately evaluated in terms of the classical solution for $r$, say $r^{*}(\tilde{\sigma})$, which minimizes $E$. And it is approximated by the global minimum of the function $n$ with respect to $r$. For this solution, we expect $\partial_{\tilde{\sigma}} r^{*} \sim 0$ for a wide range of $\sigma$ for large $\tilde{L}$. Then we obtain

$$
E \sim \frac{n\left(r^{*}\right)}{2 \pi \alpha^{\prime}} \tilde{L}
$$

and this implies the linear potential between quark and anti-quark. For the case of small $q$, we obtain

$$
\begin{aligned}
r^{*} & =r_{0}\left(1+\frac{q}{16}+\cdots\right), \\
n\left(r^{*}\right) & =|\lambda| R^{2}\left(1+\frac{q}{12}+\cdots\right),
\end{aligned}
$$

where we notice $\lambda=-4 r_{0}^{2} / R^{4}$. After all we find the tension $\tau_{U}$ of the U-shaped string configuration as

$$
\tau_{U}=\frac{|\lambda| R^{2}}{2 \pi \alpha^{\prime}}\left(1+\frac{q}{12}+\cdots\right)
$$

As expected, the tension is finite even if $q=0$, and $\lambda$ is the essential factor to obtain the linear potential or the quark confinement. The relation of (29) is explicitly shown by the numerical calculation. 
From the Lagrangian (20), we find the following relation,

$$
e^{\Phi / 2} \frac{1}{\sqrt{(r / R)^{4} A^{2}(r)+(d r / d \tilde{\sigma})^{2}}}\left(\frac{r}{R}\right)^{4} A^{3}(r)=h,
$$

where $h$ denotes a constant of motion. Taking as $h=\left.e^{\Phi / 2}\left(\frac{r}{R}\right)^{2} A^{2}(r)\right|_{r_{m i n}}$, we get

$$
\begin{aligned}
& \tilde{L}=2 R^{2} \int_{r_{\text {min }}}^{r_{\max }} d r \frac{1}{r^{2} A(r) \sqrt{e^{\Phi(r)} r^{4} A(r)^{4} /\left(e^{\Phi\left(r_{\text {min }}\right)} r_{\text {min }}^{4} A\left(r_{\text {min }}\right)^{4}\right)-1}}, \\
& E=\frac{1}{\pi \alpha^{\prime}} \int_{r_{\text {min }}}^{r_{\max }} d r \frac{A(r) e^{\Phi(r) / 2}}{\sqrt{1-e^{\Phi\left(r_{\text {min }}\right)} r_{\text {min }}^{4} A\left(r_{\text {min }}\right)^{4} /\left(e^{\Phi(r)} r^{4} A(r)^{4}\right)}}
\end{aligned}
$$

Figure 1 shows the dependence of the energy $E$ on the distance $\tilde{L}$ at finite cosmological

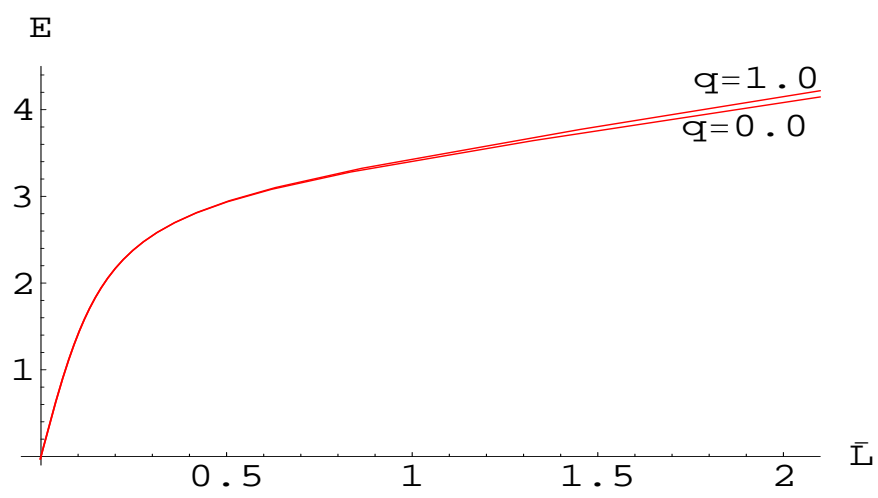

Fig. 1: Plots of $E$ vs $\tilde{L}$ at $q=1$ and $q=0$ for $\lambda=-4, R=1\left(\mathrm{GeV}^{-1}\right), r_{\max }=$ $10\left(\mathrm{GeV}^{-1}\right)$ and $\alpha^{\prime}=1\left(\mathrm{GeV}^{-2}\right)$.

constant $\lambda(=-4)$ for $q=1$ and $q=0$. In both cases, we find the linear potential and the tension given by the Eq. (32) is assured. We should notice that this confinement is realized even if $q=0$, so the gauge interactions are not essential in this case.

Here we notice that the parameter $r_{\min }$ is introduced instead of $h$, defined in (34), in the above calculation. When $r_{\text {min }}$ approaches to $r_{0}, f\left(r_{\text {min }}\right) \equiv e^{\Phi\left(r_{\text {min }}\right)} r_{\text {min }}^{4} A\left(r_{\text {min }}\right)^{4}$ approaches to its minimum value. For small $q$, it is realized at

$$
r=r_{0}(1+q / 16+\cdots) \equiv r_{M}
$$

Then, for $r_{\text {min }}=r_{M}, \tilde{L}$ diverges as

$$
\tilde{L} \sim-\left.\frac{2 R^{2}}{r_{M}^{2} A\left(r_{M}\right)} \sqrt{\frac{2 f\left(r_{M}\right)}{f^{\prime \prime}\left(r_{M}\right)}} \log \left(r-r_{M}\right)\right|_{r \rightarrow r_{M}}
$$

Therefore, it is enough to consider $r_{\min }$ in the range of $r_{\min }>r_{M}$ in order to see the behavior of the U-shaped Wilson loop configuration. On the other hand, we can not 
find the U-shaped Wilson loop when we enter into the region of $r_{\min }<r_{M}$. Instead, we find an upended shaped one in which $r_{\text {min }}$ is the top of the string. So the end points go to $r=0$ not to $r_{\max }$. We do not consider this unphysical configuration. Therefore, the natural configuration is obtained when the D7 brane position $r_{\max }$ is taken as $r_{\max }>r_{\min }>r_{M}$. This point is assured in the next section.

\section{$4 \quad$ D7 brane embedding and Chiral symmetry}

The flavor quarks are introduced by embedding D7 brane(s) in the following rewritten background,

$$
\begin{aligned}
d s_{10}^{2} & =e^{\Phi / 2}\left\{\frac{r^{2}}{R^{2}} A^{2}\left(-d t^{2}+\gamma(x)^{2} a(t)^{2}\left(d x^{i}\right)^{2}\right)\right. \\
& \left.+\frac{R^{2}}{r^{2}}\left(d \rho^{2}+\rho^{2} d \Omega_{3}^{2}+\left(d X^{8}\right)^{2}+\left(d X^{9}\right)^{2}\right)\right\}
\end{aligned}
$$

where $r^{2}=\rho^{2}+\left(X^{8}\right)^{2}+\left(X^{9}\right)^{2}$. Then the induced metric for D7 brane is obtained as,

$$
\begin{array}{r}
d s_{8}^{2}=e^{\Phi / 2}\left\{\frac{r^{2}}{R^{2}} A^{2}\left(-d t^{2}+\gamma(x)^{2} a(t)^{2}\left(d x^{i}\right)^{2}\right)+\right. \\
\left.\frac{R^{2}}{r^{2}}\left(\left(1+\left(\partial_{\rho} w\right)^{2}\right) d \rho^{2}+\rho^{2} d \Omega_{3}^{2}\right)\right\},
\end{array}
$$

where we set as $X^{9}=0$ and $X^{8}=w(\rho)$ without loss of generality due to the rotational invariance in $X^{8}-X^{9}$ plane. Then, the effective D7 brane action is given as

$$
S_{\mathrm{D} 7}=-\tau_{7} \int d^{8} \xi\left(e^{-\Phi} \sqrt{-\operatorname{det}\left(\mathcal{G}_{a b}+2 \pi \alpha^{\prime} F_{a b}\right)}-\frac{1}{8 !} \epsilon^{i_{1} \cdots i_{8}} A_{i_{1} \cdots i_{8}}\right)
$$

where $F_{a b}=\partial_{a} A_{b}-\partial_{b} A_{a} . \quad \mathcal{G}_{a b}=\partial_{\xi^{a}} X^{M} \partial_{\xi^{b}} X^{N} G_{M N}(a, b=0 \sim 7)$ and $\tau_{7}=$ $\left[(2 \pi)^{7} g_{s} \alpha^{\prime 4}\right]^{-1}$ represent the induced metric and the tension of $\mathrm{D} 7$ brane respectively. The eight form potential $A_{i_{1} \cdots i_{8}}$, which is the Hodge dual to the axion, couples to the D7 brane minimally. In terms of the Hodge dual field strength, $F_{(9)}=d A_{(8)}$ [20], the potential $A_{(8)}$ is obtained.

Taking the canonical gauge, we arrive at the following D7 brane action,

$$
S_{\mathrm{D} 7}=-\tau_{7} \int d^{8} \xi \sqrt{\epsilon_{3}} \rho^{3} \gamma(x)^{3} a(t)^{3}\left(A^{4} e^{\Phi} \sqrt{1+\left(w^{\prime}\right)^{2}}-\frac{\tilde{q}}{r^{4}}\right)
$$

Then the equation of motion for $w(\rho)$ is obtained as,

$$
\begin{gathered}
\frac{w}{\rho+w w^{\prime}}\left[\Phi^{\prime}-\sqrt{1+\left(w^{\prime}\right)^{2}}(\Phi+4 \log A)^{\prime}\right] \\
+\frac{1}{\sqrt{1+\left(w^{\prime}\right)^{2}}}\left[w^{\prime}\left(\frac{3}{\rho}+(\Phi+4 \log A)^{\prime}\right)+\frac{w^{\prime \prime}}{1+\left(w^{\prime}\right)^{2}}\right]=0
\end{gathered}
$$


where prime denotes the derivative with respect to $\rho$. By solving this equation we find the embedded configuration of D7 brane. And from this solution, the quark mass, $m_{q}$, and the chiral condensate, $c=-\langle\bar{\Psi} \Psi\rangle$, are found through its asymptotic form at large $\rho$ as,

$$
w(\rho) \sim m_{q}+\frac{c}{\rho^{2}}
$$

according to the gauge/gravity correspondence. However we notice that the above asymptotic form, (42), must be modified. This is seen by expanding the Eq. (41) in terms of the power series of $1 / \rho^{2}$ by adding the power series [12], and we obtain

$$
w(\rho) \sim m_{q}+\frac{c_{0}+4 m_{q} r_{0}^{2} \log (\rho)}{\rho^{2}},
$$

This implies the chiral condensate receives quantum corrections in the limit of $\rho \rightarrow 0$ as $c=c_{0}+4 m_{q} r_{0}^{2} \log (\rho)$. In other words, the conformal invariance of the $4 \mathrm{~d}$ CFT is broken by the added chiral multiplet for the flavor quarks.

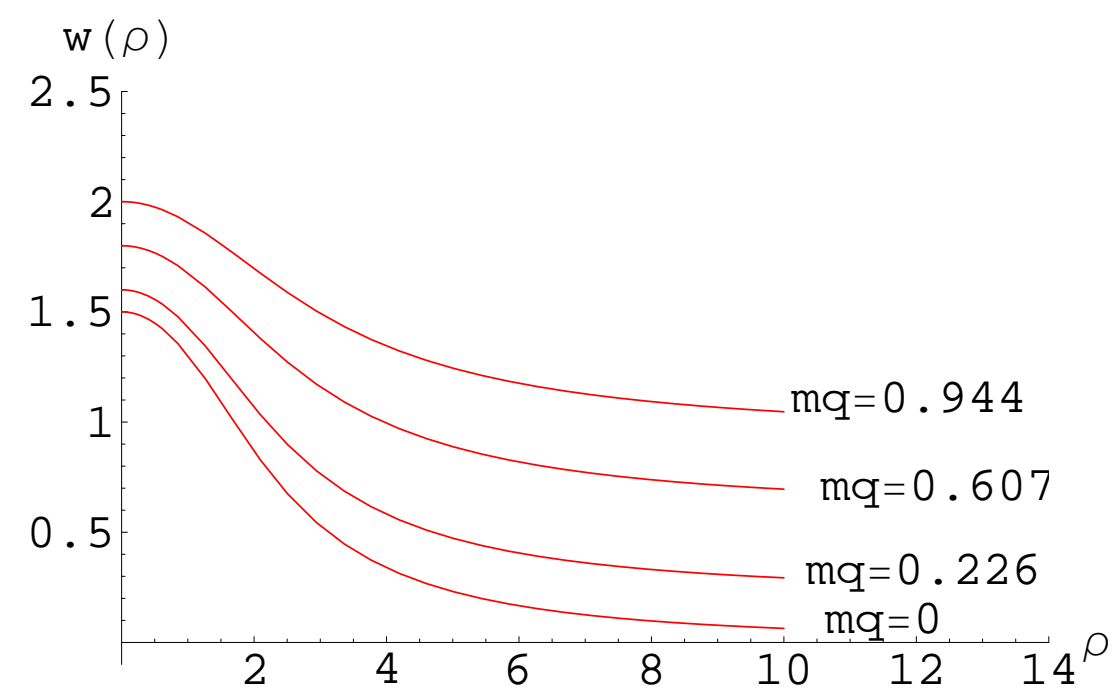

Fig. 2: Embedding solutions for $q=1.0$ and $r_{0}=1.0$. The solutions are drawn for several values of $m_{q}$, where we have taken as $R=1$.

The solutions are obtained in terms of the above asymptotic form (43), and the results are shown in the Fig. 2 for several values of $m_{q}$ in the range $0 \leq m_{q}<1$. We find $c>0$ for all the solutions. This is understood from the repulsive force between D3 and D7 branes. The potential of the D7 brane near the D3 branes is given from the D7 action as,

$$
V(w)=\tau_{7}\left(A^{4} e^{\Phi}-\frac{q}{r^{4}}-1\right)=\tau_{7}\left\{\left(1+\frac{q}{3 r_{0}^{4}}\right)\left(\frac{r_{0}}{r}\right)^{8}+\cdots\right\} .
$$

We find a strong repulsion near $r=0$. 
In the Fig. 2, the solution of $m_{q}=0$ with $c>0$, which then breaks the chiral symmetry, is shown. On the other hand, the trivial solution with $w=0$ and $m_{q}=0$, which preserves the chiral symmetry since $c=0$, also exists. The behaviors of the two solutions are very different near $\rho=0$ although they are very similar at large $\rho$. We find for the trivial solution that the energy density of the D7 brane is divergent like $1 / \rho^{5}$ at $\rho=0$ since $V \sim 1 / \rho^{8}$ near $\rho=0$. On the other hand, for the solution of $m_{q}=0$ with $c>0$, the D7 energy density approaches to zero like $\rho^{3}$ near $\rho=0$, and as a result we obtain a finite energy after $\rho$ integration in this case. Thus we can say that the chiral symmetry is spontaneously broken in the present case.

The second point to be addressed is that the solutions are far from the circle $r \sim r_{0}$. This is consistent with the stability of the fluctuations of D7 brane since the U-shaped string configurations can not be stretched up to $r=r_{0}$ as mentioned in the previous section.

\section{Possible hadron spectrum}

As shown in the previous section, the theory is in the quark confined phase and the U-shaped string configuration exists. And the tension parameter of the string configuration depends on the cosmological constant, $\lambda$, and $q$. So the meson mass also depends on these parameters and $m_{q}$. However, the $q$ dependence is rather small, and we expect that the mass spectra of the mesons are largely constrained by the geometry $\mathrm{AdS}_{4}$.

We study these point by calculating the meson mass and comparing it with the one given by the field theory in $\mathrm{AdS}_{4}$.

\subsection{Meson spectrum}

The meson spectrum is obtained by solving the equations of motion of the fields on the D7 brane. According to [23, 12], firstly we consider the fluctuations of the scalar mesons which are defined as,

$$
X^{9}=\tilde{\phi}^{9}, \quad X^{8}=w(\rho)+\tilde{\phi}^{8} .
$$

And writing the wave functions in the following factorized form,

$$
\tilde{\phi}^{k}=\varphi^{k}\left(t, x^{i}\right) \phi_{l}^{k}(\rho) \mathcal{Y}_{l}\left(S^{3}\right), \quad(k=9,8)
$$

where $\mathcal{Y}_{l}\left(S^{3}\right)$ denotes the spherical harmonic function on three dimensional sphere with the angular momentum $l$. Then we study the linearlized field equations for $\phi_{l}^{9}(\rho)$ and $\phi_{l}^{8}(\rho)$. 


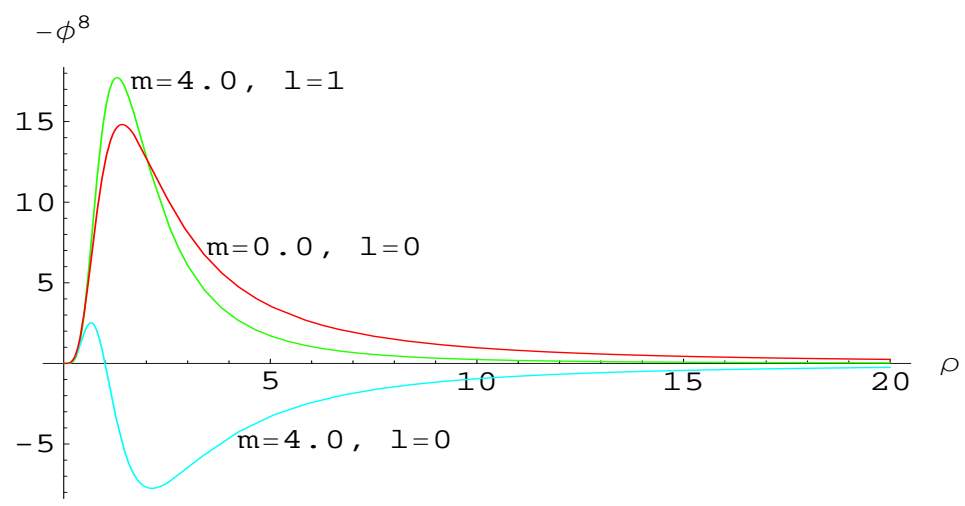

Fig. 3: The solutions for $w=q=0$. The one of (i) $m=0, l=0$, (ii) $m^{2}=-4 \lambda, l=0$ and (iii) $m^{2}=-4 \lambda, l=1$ are shown for $r_{0}=1.0$ and $R=1$, then $-\lambda=4$. The scale of the wave function for (iii) is expressed by multiplied by the extra factor $10^{-4}$ compared to the other two functions.

\subsubsection{Solution for $w=0$ and $q=0$}

At first, we consider the spectra of the mesons for a simple case where $w=0$ and $q=0$. In this case, the equations of motion for $\phi_{l}^{8}$ and $\phi_{l}^{9}$ have the following same form,

$$
\partial_{\rho}^{2} \phi_{l}+\left(\frac{3}{\rho}-\frac{8 r_{0}^{2}}{A \rho^{3}}\right) \partial_{\rho} \phi_{l}+\left[\frac{m^{2} R^{4}}{A^{2} \rho^{4}}-\frac{l(l+2)}{\rho^{2}}+\frac{8 r_{0}^{2}}{A \rho^{4}}\right] \phi_{l}=0,
$$

where $\phi_{l}$ denotes $\phi_{l}^{8}$ or $\phi_{l}^{9}$. The solution of this equation is obtained after some calculation as

$$
\begin{gathered}
\phi_{l}=\left(r_{0}^{2}+\rho^{2}\right)^{-\left(3+\sqrt{9+\bar{m}^{2}}\right) / 2}\left\{c_{1} \rho^{4+l} F\left(\alpha, \alpha+l+1, l+2,-\frac{\rho^{2}}{r_{0}^{2}}\right)\right. \\
\left.+c_{2} \rho^{2-l} F\left(\alpha, \alpha-l-1,-l,-\frac{\rho^{2}}{r_{0}^{2}}\right)\right\}, \\
\alpha=\frac{1}{2}\left(1-\sqrt{9+\bar{m}^{2}}\right), \quad \bar{m}=R^{2} m / r_{0},
\end{gathered}
$$

where $c_{1}$ and $c_{2}$ are arbitrary constants and $F$ denotes the hypergeometric function. In order to have a convergent solution $\phi$ at small $\rho$, we must take as $c_{2}=0$. And from the condition at large $\rho, \alpha$ must be set as

$$
\alpha+l+1=-n, \quad n=0,1,2, \cdots
$$

The solution is expressed by the finite power series of $\rho$ due to this condition, and we find the normalizability of the wave-function. Then we find the following result,

$$
m^{2}=-\lambda(l+n)(l+n+3) .
$$

This represents precisely the same spectrum with the one obtained by Avis, Isham and Storey [14] in the $\mathrm{AdS}_{4}$ for the scalar fields. This point is important since the 
mass scale $\lambda$ in the Eq. (49) is largely modified for the case of $w \neq 0$ and $q \neq 0$ as shown in the next subsection. In the present case, $w=0$ and $q=0$, then the quantum effect coming from the gauge interactions are all suppressed. Then the above formula is obtained.

We show the typical wave functions for $m^{2}=0$ and $m^{2}=-4 \lambda$ in the Fig. (3). The mass eigenvalue $m=4$ is obtained by the two degenerate states, $(n, l)=(0,1)$ and $(1,0)$, whose wave-functions are shown in the Fig. (3). They have the different functional forms. In the next sub-section, we can see the splitting of these degenerate mass by introducing non-zero $w(\rho)$ and $q$. They therefore deviate from the above mass formula (49).

These solutions preserve the chiral symmetry since the chiral condensate vanishes in the case of $w=0$. However, they are not realized from the energy principle since the one of $w \neq 0$ with the same boundary condition at $\rho=\infty$ is preferred, then the chiral symmetry is spontaneously broken. The mass spectra in this realistic case $w \neq 0$ are examined by the numerical analyses in the next sub-section, and we can see the details of the deviations of the meson spectra.

\subsubsection{Solution for $w \neq 0$}

Next, we get the linearlized field equations for $\phi_{l}^{9}(\rho)$ and $\phi_{l}^{8}(\rho)$ for $w \neq 0$ as follows

$$
\begin{gathered}
\partial_{\rho}^{2} \phi_{l}^{9}+\frac{1}{L_{0}} \partial_{\rho}\left(L_{0}\right) \partial_{\rho} \phi_{l}^{9}+\left(1+w^{\prime 2}\right)\left[\left(\frac{R}{r}\right)^{4} \frac{m_{9}^{2}}{A^{2}}-\frac{l(l+2)}{\rho^{2}}-2 K_{(1)}\right] \phi_{l}^{9} \\
+\left(1+w^{\prime 2}\right)^{1 / 2} \frac{1}{r} \frac{\partial \Phi}{\partial r} \phi_{l}^{9}=0 \\
L_{0}=\rho^{3} e^{\Phi} A^{4} \frac{1}{\sqrt{1+w^{\prime 2}}}, \quad K_{(1)}=\frac{1}{e^{\Phi} A^{4}} \partial_{r^{2}}\left(e^{\Phi} A^{4}\right)
\end{gathered}
$$

and

$$
\begin{gathered}
\partial_{\rho}^{2} \phi_{l}^{8}+\frac{1}{L_{1}} \partial_{\rho}\left(L_{1}\right) \partial_{\rho} \phi_{l}^{8}+\left(1+w^{\prime 2}\right)\left[\left(\frac{R}{r}\right)^{4} \frac{m_{8}^{2}}{A^{2}}-\frac{l(l+2)}{\rho^{2}}-2\left(1+w^{2}\right)\left(K_{(1)}+2 w^{2} K_{(2)}\right)\right] \phi_{l}^{8} \\
+\left(1+w^{\prime 2}\right)^{3 / 2}\left[\left(2 r K_{(1)} \frac{\partial \Phi}{\partial r}+\frac{\partial^{2} \Phi}{\partial r^{2}}\right) \frac{w^{2}}{r^{2}}+\frac{\partial \Phi}{\partial r} \frac{\rho^{2}}{r^{3}}\right] \phi_{l}^{8} \\
=-2 \frac{1}{L_{1}} \partial_{\rho}\left(L_{0} w w^{\prime} K_{(1)}\right) \phi_{l}^{8} \\
L_{1}=\frac{L_{0}}{1+w^{\prime 2}}, \quad K_{(2)}=\frac{1}{e^{\Phi} A^{4}} \partial_{r^{2}}^{2}\left(e^{\Phi} A^{4}\right) .
\end{gathered}
$$

Where four dimensional mass $m_{9}$ and $m_{8}$ are defined by

$$
-\square_{4} \varphi^{k}=\ddot{\varphi}^{k}+3 \frac{\dot{a}}{a} \dot{\varphi}^{k}-\frac{1}{a^{2} \gamma^{3}} \partial_{i}\left(\gamma \partial_{i} \varphi^{k}\right)=-m_{k}^{2} \varphi^{k} . \quad(k=9,8)
$$


In deriving the above equations (50) of $\phi_{l}^{9}$ and (52) of $\phi_{l}^{8}$, we used

$$
r^{2}=\rho^{2}+\left(\phi_{l}^{8}\right)^{2}+\left(\phi_{l}^{9}\right)^{2}+w^{2}+2 w \phi_{l}^{9}
$$

But we should notice here that the variable $r$ in the above field equations is understood as $r^{2}=\rho^{2}+w^{2}$ since we are considering the linearlized equations.

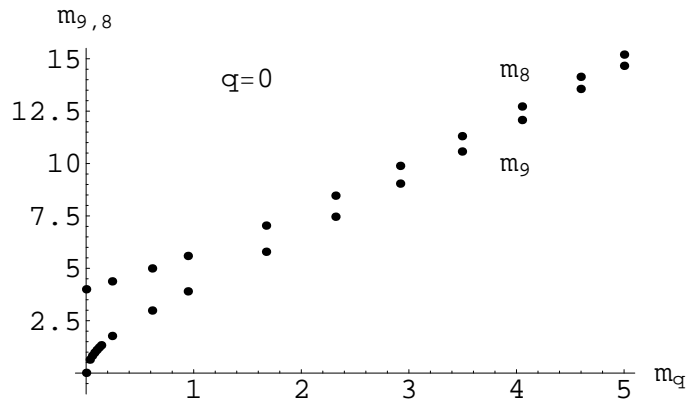

(a)

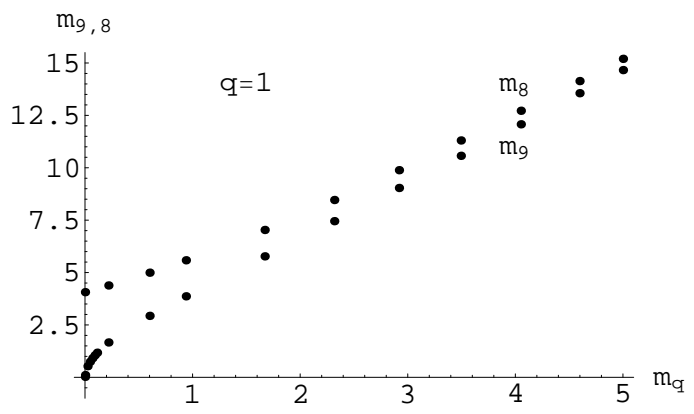

(b)

Fig. 4: $m_{9,8}$ vs $m_{q}$ for $l=n=0, r_{0}=1.0$ and $R=1$ and (a) $q=0$, (b) $q=1$.

In Figs. 4(a), 4(b), the numerical results of the mass eigenvalues, $m_{9}$ and $m_{8}$, are plotted as functions of $m_{q}$. These values are all for the nodeless solutions, i.e. for the lowest mass states. It seems that they approach to the same values at large $m_{q}[23]$. On the other hand, as seen from Figs. 5(a), 5(b), we have verified that the relation $m_{9}^{2} \propto m_{q}$ holds very accurately in the small $m_{q}$ region for both $q=0$ and $q=1$. This fact establishes that $\phi_{l=0}^{9}$ is the Nambu-Goldstone boson as expected and implies that some kind of relation like GellMann-Oakes-Renar.

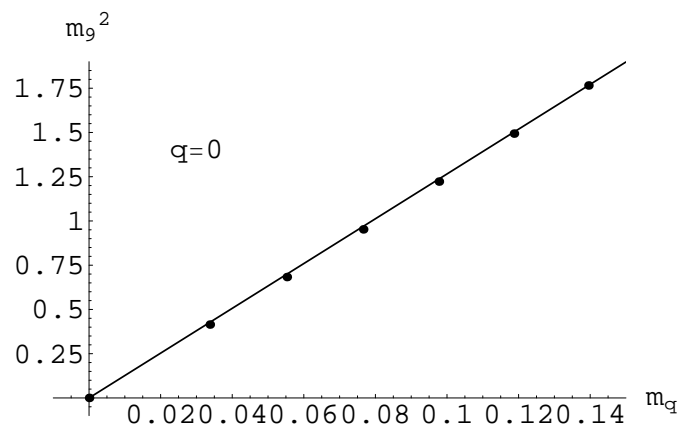

(a)

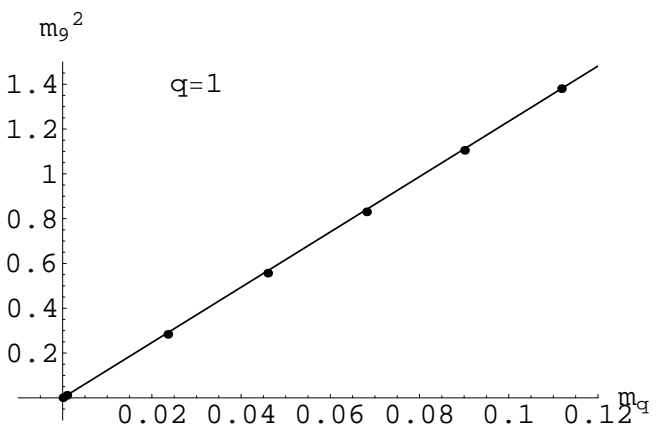

(b)

Fig. 5: $m_{9}^{2}$ vs $m_{q}$ for $l=n=0, r_{0}=1.0$ and $R=1$ and (a) $q=0$, (b) $q=1$.

Next, let us compare our numerical results with the mass formula of scalars given in $\mathrm{AdS}_{4}$ by Avis, Isham and Storey [14]. In the Ref. [14], the masses of scalars in $\mathrm{AdS}_{4}$ are given

$$
m_{I}^{2}=K(I-3) I, \quad I>2
$$


where $m_{I}$ is written for the case without the conformal coupling of the scalar and the gravity, and $I$ is given by (i) $2<I<5 / 2$ or (ii) $I=3,4,5, \ldots$. The case (i) represents continuous spectrum while the case (ii) does discrete one. The parameter $K$ is related to our $\lambda$ as,

$$
K={ }^{(4)} R / 12=-\lambda,
$$

where ${ }^{(4)} R$ denotes four dimensional scalar curvature.
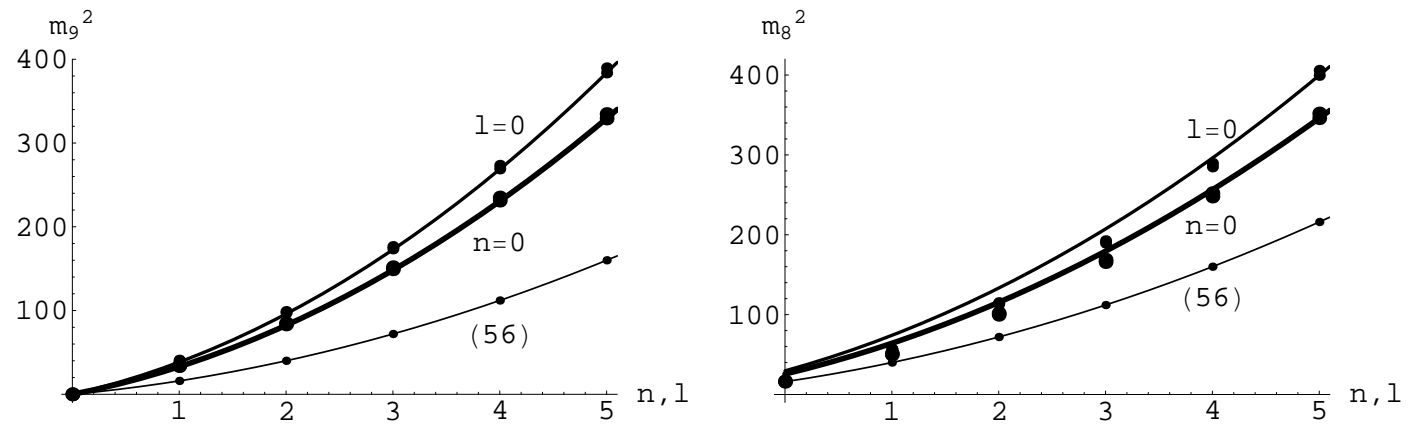

(a) $m_{9}^{2}$ vs $n, l$ at $m_{q}=10^{-5}$ with $r_{0}=1.0$, (b) $m_{8}^{2}$ vs $n, l$ at $m_{q}=10^{-5}$ with $r_{0}=1.0$, $R=1$ and $\lambda=-4 \quad R=1$ and $\lambda=-4$

Fig. 6: Small points and the thin curve corresponds to the formula (56) . Mid-large points and the mid-thick curve represent the obtained values and a fitted curve, respectively, for node $(n)$ excitations with $l=0$ while large points and thick curve represent obtained values and a fitted curve, respectively, for $l$-excitations with $n=0$.

Let us begin with eigenvalues at $m_{q}=10^{-5}$ shown in Fig. 6. Since obtained eigenvalues have zero or finite nodes (denoted by $n$ ) or integer $l$, they correspond to the case (ii) of the mass formula (56). The continuous spectrum (i), which corresponds to tachyons, does not exist in our case. It seems somewhat curious from the viewpoint of AdS/CFT correspondence that the corresponding spectrum to (i) does not exist. And we consider that the ground state for $m_{9}^{2}$ corresponds to $I=3$ since it is massless while the ground state for $m_{8}^{2}$ corresponds to $I=4$ since it is massive. Obtained values are very good agreement with the formula (56) for the ground states. Their values are $m_{9}^{2}=1.31 \times 10^{-4}$ for $q=0$ and $1.12 \times 10^{-4}$ for $q=1$ while $m_{8}^{2}=16.0$ for $q=0$ and 16.5 for $q=1$.

However, the numerical results deviate from the mass formula for the exited states with respect to $n$ and $l$. We can see that the larger $n(l)$ with $l=0(n=0)$, the deviations becomes larger from the formula (56). These deviations might be reduced to the gauge interactions between quark and anti-quark which make a bound state. As shown in the previous sub-section, the formula (56) is reproduced when we set $w=0$. On the other hand, the mass deviates from this formula when we take into account of non trivial $w$. Since the profile function $w$ includes the quark mass and the vacuum expectation value of $\bar{\Psi} \Psi$ and other information of the interaction between the quarks and the gauge fields. Then, the deviation observed is naturally understood as 


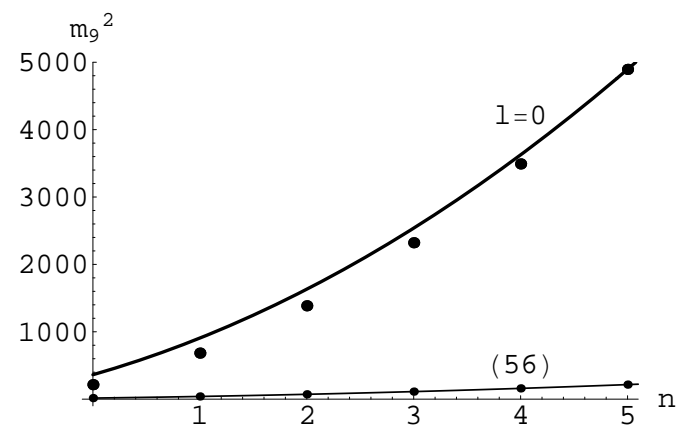

(a) $m_{9}^{2}$ vs $n$ at $m_{q}=5.0$ with $r_{0}=1.0, R=1$ and $\lambda=-4$

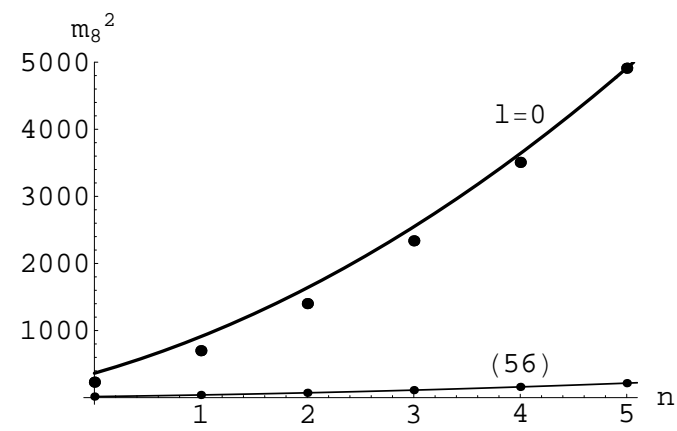

(b) $m_{8}^{2}$ vs $n$ at $m_{q}=5.0$ with $r_{0}=1.0, R=1$ and $\lambda=-4$

Fig. 7: Points and the curve represent the obtained values and a fitted curve, respectively, for node $(n)$ excitations with $l=0$. The lower lying points and the thin curve correspond to the mass formula (56).

the reflection from the gauge interactions to the meson mass. We can see this effect through the change of the mass scale in the mass formula as shown below.

Fortunately obtained values are almost degenerate for $q=0$ and $q=1$ as seen from Fig. 6. So, we attempt to fit the data at the present stage by changing $K$ by hand such that the obtained values for the highest excited states with $q=0$ are reproduced. The purpose to fit in such a way is as follows; since obtained values for ground states are in good agreement with the mass formula (56) and deviate for higher excited states, we want to know conversely deviations for lower lying states when the above mentioned fitting are performed. The results are as follows for $n$-excitations with $l=0$,

$$
\begin{array}{ll}
K_{n}^{(0)}=2.40 K, & \text { for } m_{9}^{2}, \\
K_{n}^{(0)}=1.85 K, & \text { for } m_{8}^{2},
\end{array}
$$

and for $l$-excitations with $n=0$,

$$
\begin{array}{ll}
K_{l}^{(0)}=2.06 K, & \text { for } m_{9}^{2}, \\
K_{l}^{(0)}=1.60 K, & \text { for } m_{8}^{2} .
\end{array}
$$

The coincidence is very (rather) good for $m_{9}^{2}\left(m_{8}^{2}\right)$ so that we may conclude that $I$ dependence of the formula (56) is trustable. But it should be noted that $n$-excitations and $l$-excitations is split because $K_{n}^{(0)} \mathrm{s}$ and $K_{l}^{0} \mathrm{~s}$ take different values. Although the mass formula (49) is degenerate for $n$-excitations and $l$-excitations, it is obtained for $w=0$, which is not the real solution. The real solution is $w \neq 0$ for $m_{q}=0$ and the formula (49) does not contradict with the splitting mentioned above.

Next we study the case of large $m_{q}$. In the case of $m_{q}=5.0$, obtained values are rather large compared with the ones given by the mass formula (56) as shown in Fig. 7. This is because $K=-\lambda$ is independent of $m_{q}$ whereas we interpret it should be changed as $K_{(n, l)} \rightarrow \tilde{K}_{(n, l)}\left(\lambda, m_{q}, g_{\mathrm{YM}}^{2}, \ldots\right)$. But the definite and detailed form of 
$\tilde{K}$ is unknown at the present stage so that we again choose the fitting by changing $K$ by hand as $m_{q}=10^{-5}$ case. The results are as follows for $n$-excitations with $l=0$,

$$
\begin{array}{ll}
K_{n}^{(5)}=22.7 K, & \text { for } m_{9}^{2}, \\
K_{n}^{(5)}=22.7 K, & \text { for } m_{8}^{2} .
\end{array}
$$

The coincidences are relatively good so that we may again conclude that $I$-dependence of the formula (56) is trustable. To derive more realistic mass formula is a future problem.

\subsection{Baryon}

It has been shown that baryons correspond to D5-branes wrapped around the compact manifold $M_{5}$ [24, 25]. Here we assume it to be $S^{5}$. The brane action of such a D5 probe is

$$
S_{\mathrm{D} 5}=-\tau_{5} \int d^{6} \xi e^{-\Phi} \sqrt{\mathcal{G}},
$$

where $\left(\xi_{i}\right)=\left(X^{0}, X^{5} \sim X^{9}\right), \tau_{5}$ represents the tension of D5 brane, and $\mathcal{G}=-\operatorname{det}\left(\mathcal{G}_{i, j}\right)$ for the induced metric $\mathcal{G}_{i j}=\partial_{\xi^{i}} X^{M} \partial_{\xi^{j}} X^{N} G_{M N}$. The mass of the wrapped D5-brane is then

$$
M_{\mathrm{D} 5}(r, \lambda)=\tau_{5} e^{-\Phi} \sqrt{\mathcal{G}}=\tau_{5} \pi^{3} R^{4} r A(r) e^{\Phi / 2} .
$$

Before seeing the $\lambda$ dependence of this quantity, we consider the case of $\lambda=0$,

$$
M_{\mathrm{D} 5}(r, 0)=\tau_{5} \pi^{3} R^{4} r \sqrt{1+\frac{\tilde{q}}{r^{4}}} .
$$

where $\tilde{q}$ is given by (12). This has a global minimum at $r=r_{\min }=\tilde{q}^{1 / 4}$. Its value is given as $M_{\mathrm{D} 5}\left(r_{\min }\right)=\tau_{5} \pi^{3} R^{4}(4 \tilde{q})^{1 / 4}$, and this is regarded as the baryon mass here. In the $4 \mathrm{~d}$ Minkowski limit, namely at $\lambda=0$, the baryon mass is induced by the $\tilde{q}$, i.e. by the gauge-field condensate.

In the presence of $\lambda$, namely in $\mathrm{AdS}_{4}$, the minimum of $M_{\mathrm{D} 5}$ appears even if $q=0$. For $q=0$, we obtain $M_{\mathrm{D} 5}\left(r=r_{0}, 0\right) /\left(\tau_{5} \pi^{3} R^{4}\right)=2 r_{0}$ as the minimum, which is realized at $r=r_{0}$.

Fig. 8(a) and (b) show the $r$ dependence of $M_{\mathrm{D} 5}(r)$ for three values of $\lambda$ and $q$ respectively. For any finite values of $\lambda$, even if $q=0$, there exist a minimum at an appropriate point of $\mathrm{r}$, and the minimum value of $M_{\mathrm{D} 5}$ increases with $\lambda$ and $q$. This point is consistent with the above analysis of mesons, whose mass increases with these parameters.

\section{Summary}

In this paper, the non-perturbative properties of the gauge theories in the $\mathrm{AdS}_{4}$ are studied in the dual supergravity by including the light flavor quarks, which are introduced by a D7 brane embedding. 

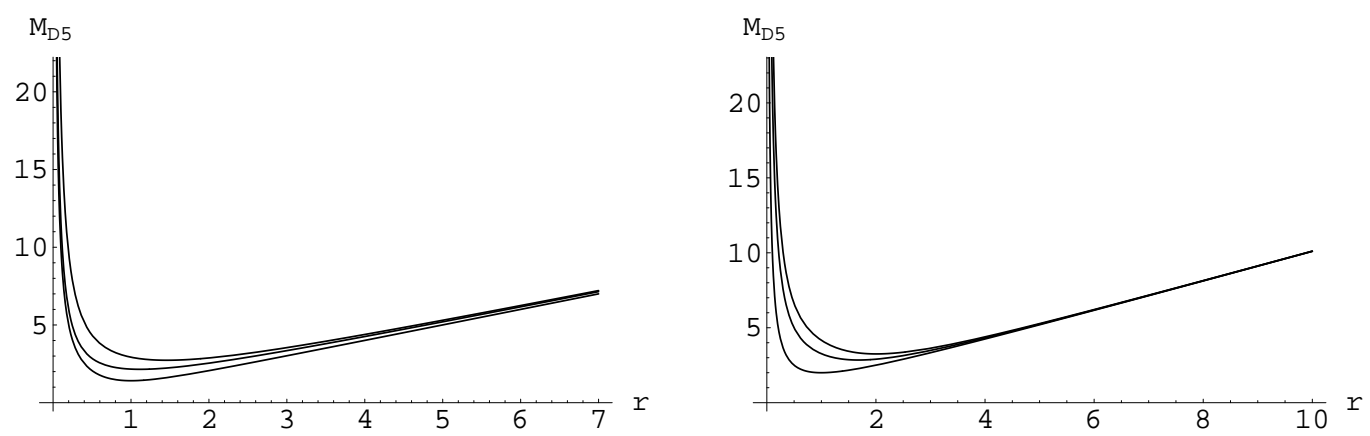

(a) The curves show $\left.M_{\mathrm{D} 5}\right|_{\lambda=0},\left.M_{\mathrm{D} 5}\right|_{\lambda=-4}$, and (b) For $\lambda=-4,\left.\quad M_{\mathrm{D} 5}\right|_{\tilde{q}=0},\left.M_{\mathrm{D} 5}\right|_{\tilde{q}=10}$, and $\left.M_{\mathrm{D} 5}\right|_{\lambda=-5.76}$, respectively, from bottom to up $\left.M_{\mathrm{D} 5}\right|_{\tilde{q}=20}$, are shown from bottom to up. for $\tilde{q}=1$.

Fig. 8: The D5-brane mass $M_{\mathrm{D} 5}(r)$ as a function of $r$ for $R=1$ and $\tau_{5}=1 / \pi^{3} R^{4}$.

In our model, in the limit of Minkowski space limit $\left(r_{0} \rightarrow 0\right)$, we have $g_{\mathrm{YM}}^{2}=1+\frac{\tilde{q}}{r^{4}}$, which is equivalent to the supersymmetric solution given previously [10]. In this limit, quark is confined due to the strong YM coupling constant in the infrared limit, $r \rightarrow 0$, for $\tilde{q}>0$. On the other hand, in the $\mathrm{AdS}_{4}$ space-time (finite $r_{0}$ ), we find $g_{\mathrm{YM}}^{2} \rightarrow 1+\frac{q}{3}$ in the infrared limit, $r \rightarrow 0$. While this coupling constant seems to be small to get the quark confinement, we find the confinement by calculating the Wilson loop which leads to the area law and the divergent effective single quark mass. These results are obtained even if $q=0$, and the important factor to get the confinement is reduced to the warp factor $A(r)$ which diverges at $r=0$ for finite $r_{0}$. In this sense, contrary to the case of the Minkowski space-time, the gauge interaction does not play any important role for the quark confinement in the $\mathrm{AdS}_{4}$ case. The important point is the geometry itself.

As for the chiral symmetry, we could expect its spontaneous breaking due to the repulsion between the D7 and the D3 branes. Actually we could find a numerical solution for the profile function $w(\rho)$, with $m_{q}=0$ and $c>0$, and it has a finite D7 energy which is obtained after $\rho$ integration. On the other hand, the trivial solution $w(\rho)=0$, the one of $m_{q}=c=0$, leads to an infinite D7 energy due to the infinite repulsion at $r=0$ due to the D3 branes. We thus find the spontaneous breaking of the chiral symmetry.

In the present case, for all the solutions of $w(\rho)$, we need a correction like $m_{q} \lambda \log (\rho)$ to the expectation value of $\langle\bar{\Psi} \Psi\rangle$. This is expected when conformal and supersymmetry is broken even in the UV limit as in the present model. Since this correction is proportional to $m_{q}$, the loop corrections of the massive hypermultiplet added to CFT would be responsible to this result.

The scalar meson spectra are calculated and we could assure the Goldstone boson due to the chiral symmetry breaking. The coincidence of the obtained masses to the mass formula given in Ref. [14] are very good for the lowest states. On the other 
hand, for the exited states, our results deviate from the mass formula. However, when we modify $K$ by hand in the mass formula (56), we find better coincidences. This is interpreted as the reflection of the gauge interaction between quark and anti-quark when they form bound states.

And baryon mass is studied by regarding it as the energy of the D5 brane, wrapped on $S^{5}$, embedded at a stable point with respect to the coordinate $r$. For any finite values of $\lambda$, even if $q=0$, we find a minimum or a stable point at an appropriate point of $r$. This is consistent with the meson case.

\section{Acknowledgments}

This work has been supported in part by the Grants-in-Aid for Scientific Research (13135223) of the Ministry of Education, Science, Sports, and Culture of Japan.

\section{References}

[1] J. M. Maldacena, Adv. Theor. Math. Phys. 2, 231 (1998) hep-th/9711200.

S. S. Gubser, I. R. Klebanov and A. M. Polyakov, Phys. Lett. B 428, 105 (1998) hep-th/9802109.

E. Witten, Adv. Theor. Math. Phys. 2, 253 (1998) hep-th/9802150]. A.M. Polyakov, Int. J. Mod. Phys. A14 (1999) 645, (hep-th/9809057).

[2] A. Karch and E. Katz, JHEP 0206, 043(2002).

[3] M. Kruczenski, D. Mateos, R.C. Myers and D.J. Winters, JHEP 0307, 049(2003).

[4] M. Kruczenski, D. Mateos, R. C. Myers and D. J. Winters, JHEP 0405, 041 (2004).

[5] J. Babington, J. Erdmenger, N. J. Evans, Z. Guralnik and I. Kirsch, Phys. Rev. D69, 066007 (2004).

[6] N. J. Evans and J. P. Shock, Phys. Rev. D70, 046002 (2004).

[7] T. Sakai and J. Sonnenschein, JHEP 0309, 047 (2003) arXiv:hep-th/0305049.

[8] C. Nunez, A. Paredes and A.V. Ramallo, JHEP 0312, 024(2003).

[9] T. Sakai and S. Sugimoto, Prog. Theor. Phys. 113(2005)843, hep-th/0412141;

Prog. Theor. Phys. 113(2005)843 hep-th/0507073.

[10] K. Ghoroku and M. Yahiro, Phys. Lett. B 604, 235 (2004).

[11] K. Ghoroku, T. Sakaguchi, N. Uekusa and M. Yahiro, Phys. Rev. D71(2005)106002. 
[12] K. Ghoroku, M. Ishihara and A. Nakamura, hep-th/0609152, to be published in Phys. Rev. D.

[13] T. Hirayama, JHEP 0606, 013(2006) hep-th/0602258).

[14] S. J. Avis and C. J. Isham and D. Storey, Phys. Rev. D18, 3565(1978).

[15] "Locally Localized Gravity", A. Karch and L. Randall, JHEP 0105 (2001) 008, [hep-th/0011156].

[16] "Higgs Phenomenon for 4-D Gravity in Anti de Sitter Space", M. Porrati, JHEP 0204 (2002) 058, [hep-th/0112166].

[17] "Supersymmetry and the AdS Higgs Phenomenon", B. A. Burrington and J. T. Liu, JHEP 0403 (2004) 059, hep-th/0311205|.

[18] A. Kehagias and K. Sfetsos, Phys. Lett. B 456, 22(1999) [hep-th/9903109].

[19] H. Liu and A.A. Tseytlin, Nucl. Phys. B553(1999)231 [hep-th/9903091].

[20] G. W. Gibbons, M. B. Green and M. J. Perry, Phys.Lett. B 370 (1996) 37, [hep-th/9511080].

[21] H. Lu, C.N. Pope and J. Rahmfeld, J. Math. Phys. 40 (1999) 4518. [hep-th/9805151].

[22] S. Gubser, hep-th/9902155].

[23] I. Brevik, K. Ghoroku and A. Nakamura, Int. J. Mod. Phys. D15 (2006) 57.

[24] D. J. Gross and H. Ooguri, Phys. Rev. D58, 106002(1998) hep-th/9805129].

[25] E. Witten, JHEP 9807, 006(1998) [hep-th/9805112]. 